\title{
Pertinence des hospitalisations posturgences de moins de 24 heures en dehors des unités d'hospitalisation de courte durée
}

\section{Appropriateness of Hospitalization from Emergency Department with Less than 24 h Length of Stay Out of Acute Emergency Care Unit}

\author{
A. Aubrion - M. Morin - E. Roupie - R. Macrez \\ Reçu le 27 juin 2018; accepté le 24 janvier 2019 \\ (C) SFMU et Lavoisier SAS 2019
}

Résumé Objectifs : L'overcrowding — ou surcharge — des services d'urgence hospitaliers est un problème de santé international, qui impacte la qualité des soins et la durée moyenne de séjour (DMS). Cette surpopulation est liée majoritairement au manque d'accès aux lits d'hospitalisation. Notre objectif principal était d'étudier la pertinence des admissions hors unité d'hospitalisation de courte durée (UHCD) ayant une DMS inférieure à 24 heures.

Méthode : Notre étude observationnelle descriptive rétrospective sur l'année 2016 concernait les patients hospitalisés hors UHCD de DMS de moins de 24 heures. La grille AEPf (Appropriateness Evaluation Protocole adaptée en France par la Haute Autorité de santé) utilisée permet de les séparer en trois groupes : hospitalisations pertinentes (gravité clinique ou soins), non pertinentes mais justifiées (organisationnelles), non pertinentes et non justifiées (hébergement). Les comparaisons étaient réalisées par test de $\mathrm{Chi}^{2}$.

Résultats : Sur 1006 admissions, selon la grille AEPf, 786 (78 \%) sont pertinentes (sévérité clinique, actes diagnostiques ou thérapeutiques, surveillance, etc.). Pour les 220 hospitalisations non pertinentes, 210 (95\%) sont justifiées par des contraintes organisationnelles (avis spécialisés,

A. Aubrion $(\bowtie) \cdot$ E. Roupie

Samu 14, CHU de Caen-Normandie,

avenue de la Côte-de-Nacre, CS 30001, F-14033 Caen, France

e-mail : aubrion-a@chu-caen.fr

A. Aubrion $\cdot$ M. Morin

Service des urgences et SMUR, centre hospitalier de Lisieux,

4, rue Roger-Aini, F-14100 Lisieux, France

E. Roupie $\cdot$ R. Macrez

UNICAEN, Normandie Université,

avenue de la Côte-de-Nacre, CS 30001, F-14033 Caen, France

\section{R. Macrez}

Département d'accueil et de traitement des urgences/Samu 14, CHU de Caen-Normandie, avenue de la Côte-de-Nacre,

CS 30001, F-14033 Caen, France actes d'imagerie, procédures). Quatre-vingt-dix-neuf (47\%) d'entre eux auraient pu rentrer à domicile si l'organisation des soins l'avait permis. Pour 83 (39\%) de ces hospitalisations non pertinentes, la structure la plus adaptée reste l'hôpital. Seulement dix ( $1 \%$ ) de ces 1006 hospitalisations sont non pertinentes et non justifiées. Le service d'hospitalisation était adéquat pour 805 patients $(80 \%)$.

Conclusion : Le taux d'hospitalisations pertinentes ou justifiées, de DMS de moins de 24 heures, depuis notre service est élevé. Il existerait quelques leviers organisationnels permettant d'éviter le recours à une hospitalisation.

Mots clés Hospitalisation pertinente $\cdot$ Hospitalisation justifiée $\cdot$ Grille AEPf-DMS 24 heures

Abstract Objectives: Overcrowding of hospital emergency departments is a worldwide health problem. Overcrowding negatively affect quality of care, length of stay, and patient outcomes. This overcrowding is mainly due to the lack of access to inpatient beds. In this project, the main objective was to study the appropriateness of unscheduled hospitalization stay from emergency department with an average length of stay less than $24 \mathrm{~h}$.

Method: We performed a retrospective, observational, and descriptive study in 2016. Patients enrolled in the study were the ones with average length of stay less than $24 \mathrm{~h}$. Patients hospitalized in an acute emergency care unit were excluded. Based on the Appropriateness Evaluation Protocol grid adapted for France, we created three groups of patients: appropriate hospitalization (clinical gravity), inappropriate but justified hospitalization (organization of patient care), inappropriate and non-justified hospitalization (social care). Results: Based on our inclusion criteria, 1,006 medical reports were analyzed. We showed that $786(78 \%)$ with a length of stay less than $24 \mathrm{~h}$ of hospitalizations were appropriate. Among the inappropriate hospitalization but justified, $210(95 \%)$ were justified by organizational limitations. Only 
$10(1 \%)$ of hospitalization was inappropriate and nonjustified and mainly driving by a social problem. The hospitalization service was adequate for 805 patients $(80 \%)$.

Conclusion: The rate of appropriate and justified hospitalization from emergency department with a length of stay less than $24 \mathrm{~h}$ (out of acute emergency care unit) is high. In order to avoid relevant but non-justified hospitalization, several organizational health cares may need to be improved.

Keywords Appropriate hospitalization - Inappropriate but justified hospitalization · Appropriateness - Evaluation protocol $\cdot$ Length of stay

\section{Introduction}

Le nombre de consultations dans les services d'urgences est en constante augmentation, quel que soit le pays ou le système de santé concerné $[1,2]$. Parallèlement, le phénomène d'engorgement (ou overcrowding) des services d'urgences est décrit depuis une dizaine d'années [3]. Ce problème de " surcharge » de nos services est souvent rapporté au problème d'afflux de patients ne justifiant pas d'une prise en charge urgente [4]. Plusieurs études ont prouvé que la présence de ces patients n'influait pas sur le temps d'attente général [5], que leur doléance initiale était souvent jugée non urgente à tort [6,7], et leur état nécessitant même parfois une hospitalisation [8]. Il est en revanche bien démontré que l'overcrowding est directement lié au taux d'occupation des lits de l'établissement [9-11], et l'attente d'un lit d'hospitalisation augmente le délai d'attente aux urgences et participe à la surcharge du service [2]. Plus cette attente est longue, plus la durée moyenne de séjour (DMS) et la mortalité augmentent $[9,12]$.

Depuis plusieurs années, le développement des prises en charge ambulatoires coordonnées entre la ville et l'hôpital s'est traduit par une augmentation du nombre de recours aux urgences et d'une réduction de la disponibilité des lits d'aval $[13,14]$. Le ministère de la Santé recommandait en 2011 la diminution des hospitalisations non pertinentes ou non justifiées pour améliorer l'efficience du système hospitalier français et la qualité des soins [15]. La Haute Autorité de santé (HAS) publiait en 2004 une revue sur la pertinence des soins hospitaliers et faisait traduire l'échelle américaine AEP (Appropriateness Evaluation Protocol) qui évalue la pertinence des admissions et des hospitalisations $[16,17]$. Elle est présentée comme un outil permettant l'optimisation des hospitalisations, en repérant celles qui ne sont pas pertinentes, afin de mettre en place des mesures correctives. Cette grille contient deux échelles distinctes. La première étudiant la pertinence des journées d'hospitalisation est la plus utilisée dans la littérature internationale. La seconde échelle utilisée dans cette étude étudie la pertinence des admissions non programmées postservice d'urgence et distingue trois types d'admissions non programmées en aval des services d'urgence : les hospitalisations pertinentes en raison de la gravité clinique ou des soins nécessaires, les hospitalisations non pertinentes mais justifiées par des contraintes organisationnelles et les hospitalisations non pertinentes ni justifiées qui correspondent à des hébergements sans nécessité de soins. Cet outil permet aux établissements de santé de s'évaluer et d'améliorer leurs organisations pour diminuer le nombre d'hospitalisations non pertinentes. Nous avons étudié les hospitalisations de moins de 24 heures en aval des urgences hors de l'unité d'hospitalisation de courte durée (UHCD) afin de savoir si elles étaient pertinentes ou justifiées et si des améliorations devaient être envisagées pour diminuer celles qui ne l'étaient pas.

\section{Matériels et méthodes}

Cette étude monocentrique, rétrospective et descriptive était réalisée en 2016 dans un centre hospitalier général, dont le service d'urgence recevait, en 2016, 31000 patients âgés de plus de 16 ans, dont 26,7\% étaient hospitalisés. Les durées moyennes de prise en charge dans le service des urgences étaient : 3 heures 25 de l'arrivée au départ, 2 heures 15 pour l'attente d'un scanner, 1 heure 23 pour celle de son interprétation, 1 heure 18 pour l'attente d'un lit d'hospitalisation après conclusion du dossier médical. Les patients de plus de 16 ans hospitalisés en aval du service des urgences étaient inclus si leur durée globale de séjour dans notre établissement, incluant le passage aux urgences, était inférieure à 24 heures. Les patients décédés, transférés, sortis contre avis médical ou fugueurs après hospitalisation étaient inclus. Les patients admis à l'UHCD étaient exclus (2 030 en 2016). Les données étaient recueillies après requête du département d'information médicale (DIM). Les comptes rendus médicaux de passage aux urgences et d'hospitalisation étaient analysés par un binôme de médecins urgentistes (MM, AA) préalablement formés aux grilles de l'AEPf grâce à son guide d'utilisation de la HAS. L'hospitalisation était jugée pertinente en présence de critères de sévérité clinique ou liés aux soins délivrés. En leur absence, elle pouvait être cependant considérée comme pertinente si un spécialiste d'organe avait décidé l'hospitalisation ou si la décision reposait sur des recommandations de bonne pratique mentionnées dans le dossier. Les hospitalisations non pertinentes pouvaient être qualifiées de justifiées si l'état des patients nécessitait d'autres procédures, soins ou surveillance que ceux liés à la sévérité. L'hospitalisation n'était ni pertinente ni justifiée en l'absence de ces critères. L'adéquation de la spécialité du service d'hospitalisation du patient était jugée 
et renseignée par le médecin réalisant son orientation. La CNIL donnait son accord le 17 février 2017.

\section{Analyse statistique}

Les séjours inférieurs à un jour étaient sélectionnés en comparant la date d'entrée et de sortie de l'établissement. Les critères non retrouvés dans le dossier étaient considérés comme absents. Les données étaient saisies sur le logiciel Microsoft Excel (2016). L'analyse des données était réalisée sous R version 3.4.1 du 30 juin 2017 (The R Project for Statistical Computing, Auckland, Nouvelle-Zélande). L'âge était défini comme une variable qualitative par intervalles de dix années. Les résultats étaient décrits en fréquence observée selon des groupes prédéfinis. Le test de $\mathrm{Chi}^{2}$ était utilisé pour comparer les fréquences observées de deux groupes différents. Une valeur de $p$ inférieur à 0,05 était considérée comme significative.

\section{Résultats}

L'étude portait sur 1006 hospitalisations dans notre établissement après leur prise en charge aux urgences pendant toute l'année 2016. La population était âgée de 16 à 100 ans (Tableau 1), l'âge moyen était de 53 ans. Les répartitions de l'âge et du sexe des patients étaient représentatives de la population consultant dans le service d'urgences. Quatre cent quarante-neuf patients (45\%) étaient pris en charge aux urgences en heure ouvrée (hors garde). Cinquante-sept patients $(6 \%)$ avaient déjà consulté dans le mois précédent leur admission. La répartition des hospitalisations de DMS de 24 heures était homogène sur l'année. Le service d'hospitalisation était adéquat dans $810(80 \%)$ des cas. Le service d'hospitalisation choisi était mieux adapté à la prise en charge du patient lorsque celui-ci s'était présenté en heures ouvrées, par rapport à ceux accueillis en période de permanence de soins $(p<0,05)$. À leur sortie d'hospitalisation, 847 patients $(84 \%)$ rentraient à domicile, 78 patients (10\%) étaient transférés dans un autre établissement médical ou médicosocial, et $32(3 \%)$ sortaient contre avis médical (dont $n=31$ [97\%] hospitalisations pertinentes et $n=1$ [3\%] justifiée ; un tiers pour demandes de sevrage alcoolique ou prise en charge psychiatrique, un tiers pour exploration de symptômes aigus ou de douleurs). Vingt-huit patients $(3 \%)$ décédaient. La première partie de la grille AEPf met en évidence que $78 \%(n=786)$ de ces hospitalisations étaient pertinentes. Parmi ces hospitalisations, $19 \%$ $(n=151)$ sont expliquées par des critères de gravités et $91 \%$ $(n=711)$ en raison de la nécessité de soins ou d'examens (Fig. 1A). Parmi les hospitalisations qui ne répondaient pas aux critères de pertinence, $19 \%(n=41)$ ont été reclassées en hospitalisations pertinentes grâce à un avis d'expert. La deuxième partie de la grille AEPf étudiait les 220 hospitalisations dites « non pertinentes ». Parmi celles-ci, 210 (21\%) étaient pertinentes et seulement dix $(1 \%)$ n'étaient ni pertinentes ni justifiées (chutes $[n=3]$, malaises $[n=2]$, constipations $[n=2]$, migraines $[n=1]$, plaie simple $[n=1]$, institutionnalisation prévue le lendemain $[n=1])$. La moitié concernait des patients de plus de 80 ans (Fig. 1A). Ces hospitalisations étaient justifiées par l'attente d'un avis spécialisé (45\%), d'un acte thérapeutique ou diagnostique (12\%), ou bien une surveillance simple (32\%) (Fig. 1). La troisième partie de la grille AEPf a permis de rechercher le lieu idéal d'hospitalisation pour les 220 hospitalisations non pertinentes (Fig. 1C). Pour 83 patients (39\%), la structure la plus adaptée restait l'hôpital. Quatre-vingt-dix-neuf patients (47\%) auraient pu rentrer à domicile si l'organisation des soins l'avait permis (attente d'un avis spécialisé, d'un examen). Huit patients sur 210 auraient pu rentrer à domicile s'ils avaient pu bénéficier de mise en place d'aides immédiates. Enfin, 20 patients ne pouvaient pas rentrer directement à domicile mais justifiaient d'une admission dans un établissement médicosocial de type soins de suite et réadaptation ou maison de retraite. Les hospitalisations par faute d'obtention d'un examen diagnostique $(p=0,81)$ ou d'une place immédiate en établissement médicosocial $(p=0,13)$ n'étaient pas liées à l'arrivée du patient en heure non ouvrée.

\section{Discussion}

Plus des trois quarts des hospitalisations courtes posturgences hors UHCD sont pertinentes en raison de la sévérité des patients, des soins, ou du potentiel évolutif. Toutefois, parmi le quart restant, les hospitalisations non pertinentes mais justifiées par des contraintes organisationnelles internes ne sont pas rares (une tous les deux jours) et pourraient être évitées si des actions d'amélioration étaient mises en place. Notre étude évaluait la pertinence d'hospitalisations posturgences dans notre établissement en ciblant les patients très précocement sortants. Le choix de réaliser cette étude sur une année entière permet de s'affranchir du biais de sélection lié à la fluctuation saisonnière de fréquentation d'un service d'urgences. L'analyse rétrospective permettait de ne pas influencer la collecte des critères AEPf dans les dossiers médicaux. L'âge moyen de la population était de 53 ans, en deçà des études similaires (moyennes : 57 et 68 ans) [18-20]. Cependant, ces études s'intéressaient à toutes admissions non programmées, alors que notre étude portait uniquement sur celles de DMS de moins d'une journée. Les patients les plus âgés sont généralement hospitalisés plus longtemps du fait de l'importance de leurs (poly)-pathologies et de raisons sociales. 


\begin{tabular}{|c|c|}
\hline Caractéristiques & $n=1006(\%)$ \\
\hline \multicolumn{2}{|l|}{ Sexe } \\
\hline Hommes & $511(51)$ \\
\hline Femmes & $495(49)$ \\
\hline \multicolumn{2}{|l|}{ Tranches d'âge } \\
\hline$<30$ ans & $175(17)$ \\
\hline $31-40$ ans & $141(14)$ \\
\hline $41-50$ ans & $134(13)$ \\
\hline $51-60$ ans & $143(14)$ \\
\hline $61-70$ ans & $176(17)$ \\
\hline $71-80$ ans & $116(11)$ \\
\hline $81-90$ ans & $82(8)$ \\
\hline $91-100$ ans & $38(4)$ \\
\hline \multicolumn{2}{|l|}{ Période d'admission } \\
\hline $\begin{array}{l}\text { Heures ouvrées (journée } 9 \text { h-19 h, hors } \\
\text { week-end et férié) }\end{array}$ & $449(45)$ \\
\hline $\begin{array}{l}\text { Heures non ouvrées (nuit } 19 \text { h-9 h, } \\
\text { ou week-end ou férié) }\end{array}$ & $555(55)$ \\
\hline \multicolumn{2}{|l|}{ Diagnostic principal } \\
\hline Traumatisme & $258(26)$ \\
\hline Cardiologie & $184(18)$ \\
\hline Neurologie & $140(14)$ \\
\hline Digestif & $134(13)$ \\
\hline Infectieux & $101(10)$ \\
\hline Pneumologie & $47(5)$ \\
\hline Intoxication & $45(4)$ \\
\hline Psychiatrie et trouble du comportement & $22(2)$ \\
\hline
\end{tabular}

La grille AEP est probablement l'un des outils les plus fiables et les plus utilisés pour évaluer la pertinence des admissions [18-20]. Elle présente des critères fiables, reproductibles et objectifs dans les deux premières parties de la grille, jugeant des facteurs de gravité et de la réalisation des soins pour le patient. Le guide d'utilisation de l'outil détaille le « rattrapage » des hospitalisations non pertinentes par un avis d'expert. Afin de limiter le biais de jugement, un seul binôme de médecins urgentistes a recueilli les données. Son rôle s'est limité à recueillir les données inscrites dans le dossier médical, sans se positionner en tant qu'expert contrairement à plusieurs études [18-20]. L'avis d'expert recueilli était celui du spécialiste sollicité, ou des recommandations des sociétés savantes. Cela a pu diminuer le taux d'hospitalisations pertinentes de notre étude. Une fois la pertinence étudiée, la grille AEP établit la justification de l'hospitalisation. Un examen complémentaire ou un avis spécialisé sont des critères objectifs. En revanche, la nécessité de surveillance hospitalière peut être subjective. Dans notre étude, les critères de pertinence portant sur les soins délivrés mettent en avant les actes et la surveillance des constantes. Ces résul-

\begin{tabular}{|ll|}
\hline Tableau 1 (suite) & \\
\hline Caractéristiques & $\mathbf{n}=\mathbf{1 0 0 6}(\mathbf{\% )}$ \\
\hline Gériatrie & $19(2)$ \\
Urologie & $16(2)$ \\
Hématologie & $9(0,9)$ \\
Métabolique & $7(0,7)$ \\
Rhumatologie & $7(0,7)$ \\
Autres & $17(2)$ \\
Service d'hospitalisation & \\
Médecine & $\mathbf{5 0 5}(\mathbf{5 0 )}$ \\
Neurologie & $200(20)$ \\
Cardiologie & $159(16)$ \\
Médecine interne & $56(6)$ \\
Pneumologie & $53(5)$ \\
Gériatrie & $22(2)$ \\
Néphrologie & $14(1)$ \\
Médecine ambulatoire & $1(0,1)$ \\
Unité de soins intensifs & $\mathbf{6 9}(\mathbf{7})$ \\
Chirurgie & $\mathbf{3 8 0}(\mathbf{3 8})$ \\
Devenir après 24 heures & \\
Retour à domicile & $847(84)$ \\
Sortie contre avis ou fugue & $32(3)$ \\
Retour à domicile avec hospitalisation & $1(0,1)$ \\
à domicile & \\
Transfert dans un autre hôpital & $78(8)$ \\
Placement en psychiatrie & $5(0,5)$ \\
Transfert dans une autre structure & $15(1)$ \\
médicosociale & \\
Décès & $28(3)$ \\
\hline
\end{tabular}

tats concordent avec d'autres études [18,19]. En revanche, on observe un taux moindre d'hospitalisation liée au traitement intraveineux (Fig. 1A). Ces données étant souvent manquantes dans les dossiers médicaux, nous avons limité l'item à sa description stricto sensu (traitements intraveineux à la seringue électrique ou soluté de remplissage sur état de choc), ce qui a pu sous-estimer le nombre d'hospitalisations pertinentes. Sur notre population, les grilles de l'AEPf retrouvent un taux de pertinence d'admission de $74 \%$, puis $78 \%$ après rattrapage par avis d'expert. Ce taux est comparable aux autres études qui retrouvent de 63 à $90 \%$ de pertinence d'hospitalisations [18-23]. Les hospitalisations non pertinentes sont justifiées pour $57 \%(n=120)$ principalement par l'attente d'une procédure diagnostique ou thérapeutique pour l'aide à la prise de décision. Cela dépend principalement de l'organisation des soins (l'accès à un avis ou à un acte) ou du manque de structures adéquates pouvant accueillir le patient. L'absence de différence mise en évidence entre les admissions réalisées aux heures ouvrées ou non, sur les différents critères d'hospitalisation non pertinente de l'AEPf, peut être liée au manque de puissance. 

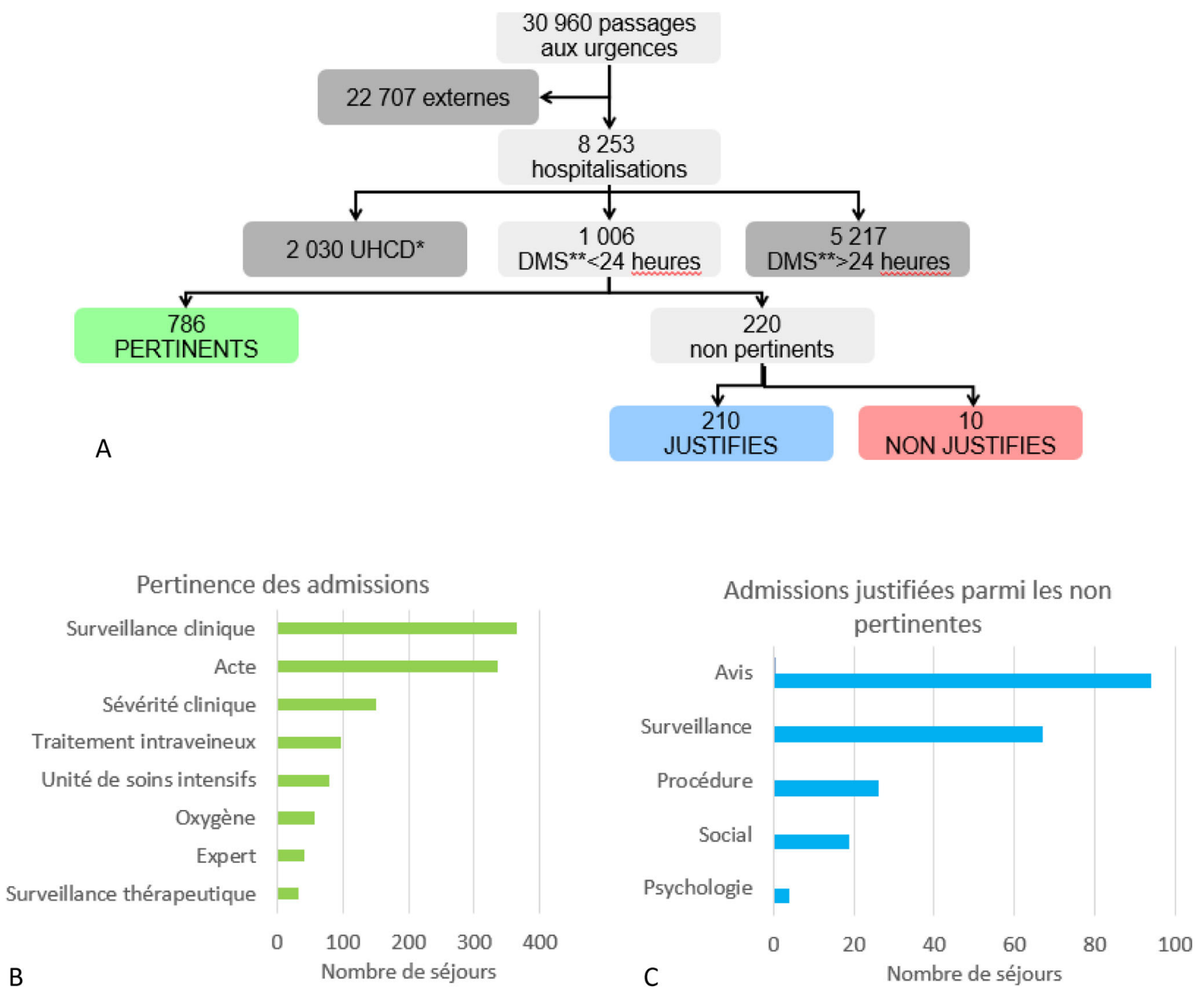

Fig. 1 Pertinence des admissions décidées aux urgences. A. Diagramme de flux. B. Pertinence des hospitalisations. C. Hospitalisations justifiées parmi les non pertinentes. *UHCD : unité d'hospitalisation de courte durée. **DMS : durée moyenne de séjour

La grande majorité des sorties contre avis étaient issues d'hospitalisations pertinentes ( $n=31$ [96,8\%]). Un tiers de ces séjours étaient motivés par des demandes de sevrage alcoolique ou de prise en charge psychiatrique, terrains d'observance plus difficile. Un deuxième tiers était lié à l'exploration de symptômes aigus ou de douleurs, qui se sont potentiellement amendés pour expliquer ce refus de soins. L'expression du refus de poursuite d'une hospitalisation pourtant jugée pertinente entraîne le raccourcissement d'un séjour qui aurait nécessité des explorations ou actes supplémentaires. De ce fait, on peut estimer que le nombre de sorties contre avis est surreprésenté sur des séjours de moins de 24 heures vis-à-vis du reste des hospitalisations. Par ailleurs, l'isolement social rend difficile le retour à domicile précoce du patient après sa prise en charge aux urgences. Celui-ci peut conduire plus fréquemment à une hospitalisation, en particulier chez les patients âgés [24-26]. En revanche, un environnement social adapté permet un rétablissement plus précoce et un retour à domicile plus rapide chez ces patients âgés [27].

Ces résultats sont à interpréter selon les limites de cette étude. Il s'agit d'une étude rétrospective fondée sur les données renseignées dans les dossiers médicaux des patients pris en charge aux urgences. Toute information non consignée étant considérée comme un critère absent, les résultats de pertinence d'hospitalisation ont pu être diminués par certains comptes rendus peu exhaustifs. Cette étude monocentrique apporte des résultats à validité externe limitée. Mais la démarche de recueil et les pistes d'amélioration de pratiques qu'elle permet d'amorcer se veulent reproductibles pour tout établissement doté d'un service d'urgence.

Ces résultats suggèrent que l'amélioration de la disponibilité des avis spécialisés ou des examens d'imagerie, par des 
actions internes ou externes au service d'urgence, pourrait diminuer le nombre d'hospitalisations non pertinentes. Parmi les actions internes, l'évaluation de la pertinence des demandes d'examens radiologiques pourrait améliorer leur disponibilité en limitant à terme les examens aux seuls pertinents. La création d'un secteur d'imagerie dédié aux urgences permettrait probablement de diminuer le temps d'attente pour ces examens. La DMS actuelle de 27 heures de l'UHCD correspond à la définition de son activité cible. Son capacitaire flottant facilite son recours et pourrait être amélioré par l'extension de ses locaux. Parmi les actions externes au service, la transformation de l'astreinte opérationnelle de radiologie ou de spécialité médicale en garde pourrait permettre d'augmenter la disponibilité des examens et avis en horaire de permanence de soins. Cependant, celleci nécessiterait de disposer d'un effectif supplémentaire de praticiens de chaque spécialité afin de permettre le maintien de la continuité des soins dans leur service respectif. De plus, si avancer ces examens au jour même permet d'éviter une hospitalisation courte (non pertinente mais justifiée), ces patients seront amenés à attendre sa réalisation dans le service des urgences, augmentant paradoxalement l'overcrowding du service [28]. Dans notre structure sur l'année 2016, 94 patients (deux par semaine) sont restés hospitalisés en attente d'un avis spécialisé conditionnant leur sortie. La pratique actuelle des avis spécialisés paraît donc adaptée. L'accès à des plannings de consultations posturgences spécialisées et de chirurgie ambulatoire permettrait de limiter l'hospitalisation de certains patients. D'autres pistes sont à explorer pour faciliter le retour à domicile des personnes fragiles, avec un accès simplifié à l'hospitalisation à domicile, aux services de soins infirmiers à domicile et la mise en place régionale de protocole d'aides au retour au domicile. Une étude s'intéressant au rôle de l'isolement du patient dans la justification de son hospitalisation pourrait également apporter des pistes de réflexion supplémentaires.

\section{Conclusion}

Dans notre établissement, $78 \%$ des hospitalisations posturgences de moins de 24 heures hors UHCD sont pertinentes (sévérité du patient, soins ou surveillance), $21 \%$ sont justifiées (avis spécialisé, acte complémentaire, cause sociale), et $1 \%$ sont jugées « non pertinentes et non justifiées » selon la grille AEPf. Ce recueil ciblé permet d'apprécier quelques pistes visant à améliorer la prise en charge de nos patients.

Conflit d'intérêt : les auteurs déclarent ne pas avoir de lien d'intérêt.

\section{Références}

1. Caillère N, Caserio-Schönemann C, Fournet N, et al (2011) Surveillance des urgences - Réseau OSCOUR ${ }^{\circledR}$ (Organisation de la surveillance coordonnée des urgences) - Résultats nationaux 2004/2011. Institut de veille sanitaire, Saint-Maurice. http:// invs.santepubliquefrance.fr/Dossiers-thematiques/Veille-et-alerte/ Surveillance-syndromique-SurSaUD-R/Reseau-OSCOUR-R (Dernier accès le 14 novembre 2018)

2. Moskop JC, Sklar DP, Geiderman JM, et al (2009) Emergency department crowding, part 1 - concept, causes, and moral consequences. Ann Emerg Med 53:605-11

3. American College of Emergency Physicians (2008) Emergency department crowding: high-impact solutions, task force report on boarding, http://www.edbenchmarking.org/assets/docs/hottopics/ 2008boardingreportcme\%201.pdf (Dernier accès le 25 avril 2018)

4. Mesnier T (2018) Assurer le premier accès aux soins, organiser les soins non programmés dans les territoires, rapport parlementaire. http://solidarites-sante.gouv.fr/ministere/documentation-etpublications-officielles/rapports/sante/article/rapport-assurer-lepremier-acces-aux-soins-organiser-les-soins-non-programmes (Dernier accès le 22 juin 2018)

5. Schull MJ, Kiss A, Szalai JP (2007) The effect of low-complexity patients on emergency department waiting times. Ann Emerg Med 49:257-64

6. Raven MC, Lowe RA, Maselli J, Hsia RY (2013) Comparison of presenting complaint vs discharge diagnosis for identifying "nonemergency" emergency department visits. JAMA 309:1145-53

7. Durand AC, Gentile S, Devictor B, et al (2011) ED patients: how nonurgent are they? Systematic review of the emergency medicine literature. Am J Emerg Med 29:333-45

8. Vertesi L (2004) Does the Canadian emergency department triage and acuity scale identify non-urgent patients who can be triaged away from the emergency department? CJEM 6:337-42

9. Guttman A, Schull MJ, Vermeulen MJ, Stukel TA (2011) Association between waiting times and short-term mortality and hospital admission after departure from emergency department: population based cohort study from Ontario, Canada. BMJ 342:d2983

10. Sprivulis PC, Da Silva JA, Jacobs IG, et al (2006) The association between hospital overcrowding and mortality among patients admitted via Western Australian emergency departments. Med J Aust 184:208-12

11. Holroyd BR, Bullard MJ, Latoszek K, et al (2007) Impact of a triage liaison physician on emergency department overcrowding and throughput: a randomized controlled trial. Acad Emerg Med 14:702-8

12. Singer AJ, Thode HC, Viccellio P, Pines JM (2011) The association between length of emergency department boarding and mortality. Acad Emerg Med 18:1324

13. Claret PG, Bobbia X, Richard P, et al (2014) Surcharge du service des urgences : causes, conséquences et ébauches de solutions. Ann Fr Med Urgence 4:96

14. Alleaume B, Boisguérin B, Bouvet M, et al (2016) Panorama, les établissements de santé - édition 2016, DREES. Collection études et statistiques, Paris, pp 10-176

15. Sanesco ${ }^{\circledR}$, Boulogne (2011) Enquête nationale pour l'évaluation des inadéquations hospitalières. Ministère de la Santé et des Sports. http://fulltext.bdsp.ehesp.fr/Ministere/Publications/2011/ $\begin{array}{llllllll}3 & 10 & 2011 & 14 & 11 & 24 & \text { INADEQUATIONS\%20HOSPITALIE- }\end{array}$ RES\%20Rapports\%20 $\overline{\%}$ Sanesco\%20090609.pdf (Dernier accès le 18 mai 2018)

16. D’Alché-Gautier MJ, Erbault M, De Vernejoul N (2004) Revue de pertinence des soins, application aux admissions et aux journées d'hospitalisation. HAS novembre 2004. https://www. 
has-sante.fr/portail/upload/docs/application/pdf/2009-08/rps guide.pdf (Dernier accès le 14 novembre 2017)

17. Gertman PM, Restuccia JD (1981) The Appropriateness Evaluation Protocol : a technique for assessing unnecessary days of hospital care. Med Care 19:855-7

18. Hodgins MJ, Moore N, Legere L (2011) Who is sleeping in our beds? Factors predicting the ED boarding of admitted patients for more than 2 hours. J Emerg Nurs 37:225-30

19. Andronikof M, Roche V, Civadier MS, et al (2011) Évaluation d'un service d'urgence possédant une unité d'hospitalisation conventionnelle. Ann Fr Med Urgence 6:85-92

20. Fournier D (2012) Évaluation de la pertinence des hospitalisations aux urgences du centre hospitalier de Chambéry. Université de Grenoble, Thèse de médecine $n^{\circ}$ 00724111. https://dumas.ccsd. cnrs.fr/dumas-00724111/document (Dernier accès le 23 juin 2018)

21. Bussy C, Hamdoud Z, Merad M, et al (2008) Évaluation de la pertinence des hospitalisations des patients consultant aux urgences de l'institut Gustave-Roussy. Risques \& qualité en milieu de soins. Thèse de médecine, université Paris-Sud, 3, pp 151-7. Accessible (uniquement en cache) sur https://webcache.googleusercontent.com/search?q=cache:5L0bjMyJFMQJ:https://www. hygienes.net/boutique/risques-qualite/evaluation-de-la-pertinencedes-hospitalisations-des-patients-consultant-aux-urgences-delinstitut-gustave-roussy $/+\& \mathrm{~cd}=1 \& \mathrm{hl}=\mathrm{fr} \& \mathrm{ct}=\mathrm{clnk} \& \mathrm{gl}=\mathrm{fr}$

22. Blanchard-Pontoizeau V (2003) Évaluation de la pertinence des hospitalisations à partir du service des urgences en fonction des critères de l'AEP (Appropriateness Evaluation Protocol). Thèse de médecine $n^{\circ} M G / 03 / 08$, université de Nantes. http://archive. bu.univ-nantes.fr/pollux/show.action?id=07765a7a-ff71-486d-95 9d-95a5523cc242 (Dernier accès le 25 avril 2018)

23. Chamelat C (2014) Évaluation de la pertinence des admissions aux urgences de l'hôpital d'instruction des armées Robert-Picqué. Médecine humaine et pathologie. Thèse de médecine, université Bordeaux-II. https://dumas.ccsd.cnrs.fr/dumas-01093454/document (Dernier accès le 14 novembre 2018)

24. Stevens TB, Richmond NL, Pereira GF, et al (2014). Prevalence of nonmedical problems among older adults presenting to the emergency department. Acad Emerg Med 21:651-8

25. Fealy GM, Treacy M, Drennan J, et al (2012) A profile of older emergency department attendees: findings from an Irish study. J Adv Nurs 68:1003-13

26. Ellis G, Marshall T, Ritchie C (2014) Comprehensive geriatric assessment in the emergency department. Clin Interv Aging 9:2033-43

27. Dickens AP, Richards SH, Greaves CJ, Campbell JL (2011) Interventions targeting social isolation in older people: a systematic review. BMC Public Health 11:647

28. Chiu IM, Yan-Ren L, Yuan-Jhen S, et al (2018) The influence of crowding on clinical practice in the emergency department. Am J Emerg Med 36:56-60 